\title{
ON RADICALS AND REPRESENTATIONS OF TOPOLOGICAL
}

\section{ALGEBRAS}

\author{
MART ABEL \\ Date of Receiving : $\quad: \quad 06.10 .2016$ \\ Date of Revision : $\quad 06.12 .2016$ \\ Date of Acceptance : $\quad 06.12 .2016$
}

\begin{abstract}
In this paper we generalize some results about Jacobson radical, topological radical and irreducible representations known for Banach algebras to the case of quite general topological algebras.
\end{abstract}

\section{Introduction}

The present paper is a continuation of the work started in [1] and [2]. Our aim is to generalize some results about the radicals and irreducible representations from the case of Banach algebras, studied in [6], to the case of general topological algebras with very few extra conditions on the algebra or its topology.

Let $\mathbb{K}$ denote either the field $\mathbb{R}$ of all real numbers or the field $\mathbb{C}$ of all complex numbers and let $A$ be an algebra over $\mathbb{K}$. As usual, we will denote by $\operatorname{Rad}(A)$ the Jacobson radical of $A$ and by $\operatorname{Inv}(A)$ the set of invertible elements of a unital algebra $A$.

By a topological algebra over the field $\mathbb{K}$ we will mean a topological vector space over the field $\mathbb{K}$, in which there is also defined the multiplication, which turns the space into an algebra and which is separately continuous in the topology of the topological vector space.

2000 Mathematics Subject Classification. Primary 16N20; Secondary 16Nxx, 46H15.

Key words and phrases. Jacobson radical; topological radicals; (anti)representations of topological algebras.

The research was supported by institutional research funding IUT20-57 of the Estonian Ministry of Education and Research. The author would like to thank the referee for suggestions which led to the improvement of the present paper.

Communicated by. Lourdes Palacios

${ }^{\dagger}$ Corresponding author. 